\title{
¿Cómo se enfrentan tres culturas corporativas en una joint venture? Caso Intesa-Venezuela
}

Berlin, Margalit*

\section{Resumen}

Este es un estudio de caso realizado en Intesa, una empresa conjunta o joint venture. En Intesa coinciden tres tipos de gerentes, según el tipo de organización de la cual provienen: de Petróleos de Venezuela (PDVSA), del sector privado nacional y del sector multinacional. Mientras los gerentes se adaptan a las culturas corporativas de estos tres sectores, traen ciertas características culturales que los diferencian entre sí. El objetivo de este trabajo es determinar si las diferencias culturales tienen una influencia en la adopción de la cultura que auspicia la empresa. Los resultados de esta investigación coinciden con los encontrados en la literatura sobre la importancia de las condiciones iniciales en las que se forma una joint venture para determinar que una cultura predomine sobre otra. Estas condiciones iniciales son: la ubicación, las instalaciones, el lenguaje utilizado en la firma, los horizontes de carrera. Este estudio muestra un elemento más que contribuye al predominio de una cultura sobre otra. Este depende de las vías que posea cada sector para transmitir su cultura corporativa, algunas son más efectivas que otras. Las menos efectivas son los talleres itinerantes, los boletines, los correos electrónicos mientras las más efectivas son los premios y sanciones que se dan en la organización.

Palabras clave: Cultura corporativa, multinacionales, gerencia, empresas conjuntas, diferencias culturales.

\section{How to Manage Three Corporate Cultures in one Joint Venture. The Intesa-Venezuela Case}

\section{Abstract}

This is a case study of Intesa, a joint company or a mixed venture. In Intesa we find three types of managers according to the company of precedence: the Venezuelan Oil Company (PDVSA), the private national sector, and the multinational sector. Since these managers are adapted to the corporate cultures of these three sectors, they bring along certain cultural characteristics that differentiate them one from another. The objective of this paper is to determine to what degree these cultural differences will influence in the adoption of the most appropriate culture for the company. The

Recibido: 00-11-28 . Aceptado: 01-11-15

Egresada de Columbia University: Bachelor of Arts, Master of Arts (Sociología) y Ph.D. (Antropología). Profesor Titular de la Universidad Simón Bolívar. E-mail: littyberlin@cantv.net 
results of this research coincide with the results found in the literature as to importance of the initial conditions in which a joint venture is organized in order to determine whether or not one culture will dominate over another. These initial conditions are: localization, installations, the language utilized in the company and the career horizon. This study shows one more element which contributed to the dominance of one culture over another. This refers to the means that each sector has at its disposal to transmit its corporate cultural, since some are more effective than others. The least effective are itinerant workshops, bulletins, and electronic mail, while the most effective are the rewards and punishments that the organization offers.

Key words: Corporate culture, multinational firms, management, joint ventures, cultural differences.

\section{Introducción}

La cultura corporativa constituye una racionalización para la conducta de las personas dentro de ella y una guía para la acción, es una cualidad que distingue una compañía de otras en el mismo ramo. Se ha observado que las empresas multinacionales auspician una cultura que les sirve para adaptarse a los distintos entornos de negocios en los cuales se insertan, mediante pautas para orientar la conducta dentro de la corporación, disminuyendo así los riesgos de insertarse en entornos de negocios muy distintos al de la casa matriz. Esos entornos, a su vez, imponen diversas exigencias a los gerentes quienes tratan de adaptarse a ellos, lo que genera conductas difíciles de predecir para las corporaciones. Mediante la aceptación de la cultura corporativa se logra que los gerentes den prioridad a las exigencias de la empresa y que muestren, conductas acordes con las metas de la firma. Sin embargo, la aceptación de la cultura corporativa depende de las oportunidades que la firma ofrece para avanzar profesionalmente (Berlin, 1994; 1996; 1999).
La cultura es un proceso de construcción social, se forma en la interacción simbólica que establecen los actores, por lo tanto es obvio que la cultura organizativa sea el resultado de esa interacción.

Compartir el sitio de trabajo con personas que provienen de distintos sectores organizacionales da lugar a la creación de estereotipos, o visiones del otro, poco objetivas, que expresan la insatisfacción que se siente por las condiciones laborales. Ello hace aún más interesante estudiar cómo las diferencias culturales son utilizadas por compañeros de trabajo insatisfechos como explicaciones de ciertos aspectos organizacionales cuando se desconoce el contexto en el cual se generan.

Un lugar apropiado para responder a la pregunta de cuál es el impacto que diferentes culturas tienen en la formación de una joint venture puede ser una empresa de este tipo en donde personas de distintas nacionalidades y de distintos sectores organizacionales (PDVSA, privado nacional y privado multinacional) se interrelacionan. Con ese propósito se buscó una joint venture formada con Petróleos de Venezuela (PDVSA). Esa joint venture fue Intesa: una empresa de infor- 
mática recién formada y de gran importancia para el país a la cual tuve acceso a través de viejas amistades 1 .

La empresa, por otra parte, estaba interesada en conocer cómo los empleados y gerentes -veían a la empresa según el sector de donde provienen: PDVSA, privado nacional o el privado multinacional. Esto indica que veían la influencia de las experiencias previamente fue adquiridas en las reacciones de los miembros a los distintos aspectos de cultura organizacional que reforzaba la alta gerencia de la empresa.

Entonces lo primero era saber qué había hecho que PDVSA (Petróleos de Venezuela, S. A.) se asociara con una empresa de informática de renombre mundial en un momento en el que los precios del petróleo habían bajado drásticamente. Se iniciaba una empresa de gran envergadura en momentos de crisis? ¿por qué? ¿qué funciones tiene ese tipo de empresa?

Algunas definiciones son, necesarias para comenzar a responder esas preguntas. Resumiendo la definición de James-Otis Rodner (1993) las joint ventures o empresas conjuntas resultan de la asociación entre dos o más empresas para los efectos de explotar un fin económico común. A menudo, pero no necesariamente, las empresas conjuntas tienen accionistas de nacionalidades diversas y son utilizadas para, por ejemplo, dos empresas mul tinacionales van a establecer una nueva compañía con el fin de explotar una actividad económica determinada.

Las joint ventures permiten compartir los costos de producción y a la vez diluir las consecuencias de una pérdida. Algunos autores tales como Contractor y Lorange (1986) y por otra parte, Rodner (1992) han notado la importancia de esta función en la industria de computación (o tecnología de información: TI). En ella los costos de fabricación son muy altos y el ciclo de vida que tiene el producto es muy corto, lo que dificulta la amortización de los costos de su desarrollo.

Las joint ventures permiten el acceso a recursos humanos tanto calificados (Rodner, 1993) como no calificados (Wong et al, 1999; Wright et al, 1998; Zeira et al, 1997) que se remuneran con salarios relativamente bajos, especialmente si vienen de países en vías de desarrollo.

En momentos de recesión, una empresa pública no puede invertir tanto como necesita en informática y para estar al día se asocia, forma una joint venture.

Por otra parte, ¿qué gana la empresa multinacional con la asociación? Una firma multinacional prefiere, por lo general, invertir sola para ganar más o para evitarse los problemas que surgen de tener un socio, pero en ciertas condiciones la co-inversión se prefiere. Algunos países consideran el ser propietarios como

1 La posibilidad de desarrollar estudios sobre culturas en organizaciones latinas está ligada al acceso que se tenga a las organizaciones formales. Mientras que en los países desarrollados es usual encontrar estudios sobre compañías identificadas con sus nombres, realizados por consultores. El acceso depende de la seguridad que se ofrezca a las organizaciones de que la información que se obtenga será utilizada en beneficio de esta y de sus miembros (Berlín, 1995). 
un importante objetivo nacional. Así, Vernon, et al (1991) notan que los países de Europa Occidental, menos inquietos acerca de la posibilidad de que algunas industrias sean extranjeras, están menos preocupados por ese asunto que otros gobiernos. India prefiere las joint ventures a las subsidiarias extranjeras. En este caso, la joint venture estudiada sirve de sede para las operaciones en Latinoamérica de una multinacional norteamericana de informática con lo cual vemos su importancia para la multinacional.

Las joint ventures ayudan a superar las reacciones xenofóbicas que han surgido muchas veces durante gobiernos nacionalistas (Killing, 1983; Vernon et al, 1991; Swan et al, 1997). Aclara Gómes Cáceres (1988), que las joint ventures necesitan de cierta apertura por parte de los gobiernos huéspedes pues, en efecto, las políticas restrictivas coinciden con una disminución de las joint ventures.

Paul Beamish (1988) en un estudio basado en entrevistas a gerentes de joint ventures en países subdesarrollados encuentra que las diferencias más importantes entre las joint ventures formadas en países desarrollados y aquellas que se establecen en un país subdesarrollado residen, por una parte, en las razones que conllevan a su creación. El motivo fundamental que conlleva a la formación del primer tipo es el conocimiento del mercado, mientras la persuasión o legislación de los gobiernos huéspedes junto con la disposición a la apertura constituyen los motivos principales del segundo tipo.

\section{Basamento teórico y metodología}

La definición de cultura corporativa que consideramos más apropiada es la de Schein (1985) y Sathe (1985) porque partimos de que ciertos aspectos de la cultura corporativa son subterráneos y, por lo tanto, son difíciles de abordar. Schein y Sathe consideran la cultura corporativa como un conjunto de patrones que generalmente no están dichos formalmente y están a nivel inconsciente. Para Schein (1985) y Sathe (1985) las observaciones no son suficientes para conocer la cultura corporativa; es necesario realizar entrevistas para indagar sobre los valores que se asumen muchas veces sin que las personas se den cuenta de ello; para entender, en otras palabras, cómo piensa la gente en lugar de qué hace la gente.

Además, partimos de que en una empresa existen diferencias entre la cultura corporativa "oficial" y la "no oficial" (Cartwright et al, 1989) que resulta de la interpretación que la mayoría le da a la cultura corporativa oficial según lo que le resulte más familiar y similar a las experiencias acumuladas a lo largo de los años así como en trabajos anteriores. $Y$ siguiendo a John Hendry (1999) en una organización pueden coexistir rasgos de una cultura jerárquica y de una cultura de mercado y estas diferencias no se ven a simple vista.

Más aún, ciertos aspectos de las culturas nacionales y/o corporativas no son fácilmente observables. Concretamente nos referimos a las dimensiones 
por medio de las cuales se describen, en este estudio, las diferencias entre las culturas corporativas que coinciden en la joint venture: el manejo del conflicto y el rechazo a la incertidumbre.

Quisimos saber más sobre el manejo del conflicto en un joint ventures, motivados por investigaciones anteriores sobre la resolución de conflictos en dos organizaciones en las que habíamos encontrado como se manejan los conflictos. El Departamento de Recursos Humanos sirve entre otras cosas para resolver los conflictos que se presentan entre el personal obrero y el personal gerencial (Berlin, 1986), y en un segundo caso se observó como en una empresa del sector privado multinacional la cultura corporativa sirve para neutralizar los conflictos que se presentan en la organización.

La tendencia a evitar la incertidumbre representa el grado en que las personas toleran la incertidumbre y la ambigüedad en diversas situaciones. Una ubicación alta en la escala de esta dimensión significa que una sociedad evitará situaciones de riesgo mediante la protección social de sus individuos y la estabilidad laboral. En países con una gran tendencia a evitar la incertidumbre como Japón, Taiwan, Hong-Kong, Venezuela, se observa una resistencia al cambio, mayor lealtad a los jefes, ascensos por edad antes que por mérito, una menor orientación hacia el logro, jerarquías claramente marcadas, preferencia por instrucciones claras y una tendencia a sospechar de los extranjeros. En países con una ubicación baja en esta dimensión tales como Estados Unidos y Singapur, se observa menos miedo al cambio, gerentes más jóvenes, mayor orientación al logro, toma de riesgos y ambición, una tolerancia al rompimiento de las reglas, mayor competencia y delegación.

El vocablo incertidumbre pasó en sociología a ser prácticamente una categoría relacionada al contexto de sociedades complejas y de riesgo. La idea de riesgo, según Anthony Giddens (1999) supone una sociedad que trata activamente de romper con su pasado- la característica fundamental, en efecto, de la civilización industrial moderna. El riesgo es la dinámica fundamental movilizadora de una sociedad volcada en el cambio que quiere determinar su propio futuro en lugar de dejarlo a la religión, la tradición o los caprichos de la naturaleza. Esta noción siempre ha estado relacionada con la modernidad.

Nos referimos aquí, primero, a la preocupación por la existencia o no de normas e instrucciones en el trabajo y, segundo, por la inestabilidad laboral. Ambas, según el análisis de Giddens (1999) forman parte del riesgo manufacturado que no sólo concierne a la naturaleza (como lo son los riesgos tradicionales) sino que penetra otras áreas de la vida.

Para su estudio la variable rechazo a la incertidumbre fue estudiada en sus dos aspectos: la necesidad de normas y descripciones que se indagó mediante las preguntas acerca de "si es posible tener todas las respuestas para ser un buen gerente" y "si se debe evitar el tener dos jefes"; y segundo, las actitudes de las personas ante la inestabilidad laboral que surgió espontáneamente de la pregunta abierta ¿A qué se deben los cambios entre la organización de la cual provienen e Intesa?" Esta manera de medir la tolerancia a la incertidumbre se deriva de la obra de Hofstede (1980), utilizada posterior- 
mente en un estudio sobre cultura en países de América Latina (Ogliastri et al, 1999).

Por otra parte, siguiendo a Guideon Kunda (1992) aprendemos que la aceptación de la cultura corporativa no es automática ni ocurre siempre que los miembros de la organización desarrollan resistencias aunque éstas sean sólo el aislamiento y la ironía (Kunda, 1992). En un estudio realizado en un banco multinacional en Caracas encontramos que la aceptación de la cultura corporativa depende de las oportunidades que la empresa ofrece a sus empleados y gerentes (Berlin, 1994, 1999). Continuando esta línea de investigación quisimos conocer qué otros factores influencian la aceptación de la cultura de la empresa.

Impacto de las diferencias culturales en las joint ventures: Algunos autores han estudiado, como señalamos en la revisión bibliográfica, el impacto de las diferencias culturales -bien sea en la incidencia de las joint ventures y en su duración (Barkema y Vermeulen, 1987; Leung et al, 1996; Park et al, 1997; Beamish, 1988), en el manejo de las joint ventures (Newman, 1992; Baird et al, 1990; Dymsza, 1988; Zeira et al, 1997; Morris et al, 1998; Kanter, 1989, 1994; Valdes Llaneza et al, 1996), en la integración cultural en una joint venture (Rodríguez,1996; 1997) y en una joint venture en formación (Carwright et al, 1989); en la transferencia de tecnología (Lam, 1996); en el ma- nejo del entorno de negocios que rodea a las joint ventures especialmente cuando se trata de países en vías de desarrollo (Zhuang et al, 1998). Más específicamente, Jane Salk (1993) sostiene que en una joint venture se tiende a la formación de sub-grupos y estereotipos culturales a pesar de los intentos de la alta gerencia por implantar una cultura oficial ${ }^{2}$.

Un aspecto que no ha sido suficientemente estudiado es el impacto de las diferencias culturales que existen entre los miembros de la organización, en la aceptación de la cultura que trata de implantar la alta gerencia de la joint venture. Este será uno de nuestros objetivos principales.

Después de analizar las tres culturas corporativas que coinciden en Intesa y la cultura que trata de implantar la alta gerencia se examinó el comportamiento de esas dos variables para cada uno de los tres sectores analizando las comparaciones que hacen los gerentes entre la empresa de donde provienen e Intesa.

Luego de analizar estas comparaciones buscando en qué se asemejan y en qué se diferencian las maneras de interpretar la cultura oficial de la empresa de cada uno de los tres grupos sociales, se analizan las posibilidades de una integración cultural en Intesa.

Así mismo revisando la literatura sobre joint ventures vemos que una característica estructural de ese tipo de empresa es su inestabilidad (Harrigan, 1986; Park et al, 1997; Salk, 1993; Killing, 1983;

2 Todos los argumentos de los autores mencionados se encuentran descritos en una versión más completa de este trabajo Berlin, M. (2000) "Cultura corporativa en una joint venture: El caso de Intesa”. Trabajo presentado para ascender a la categoría de Profesor Titular en la Universidad Simón Bolívar. 
Gómez-Cáceres, 1988; Park et al, 1996; Zeira et al, 1997; Meeker, 1988; Lei et al, 1991; Newman, 1992; Kanter, 1989; Inkpen et al, 1997).

El porcentaje de disoluciones de las joint ventures es cerca del 50 por ciento (Harrigan, 1986; Park et al, 1997; Salk, 1993). Aparte de los altos porcentajes de disolución se han detectado a través de entrevistas a gerentes otros efectos negativos como una disminución en las ganancias, fallas en la transferencia de tecnología, dificultades y problemas operacionales, desacuerdos y ansiedades por transferencia involuntaria de información de un socio a otro (Killing, 1983; GómezCáceres, 1988; Harrigan, 1986).

Gómes-Cáceres (1988) después de revisar las características de los países en donde se ubican las subsidiarias, en reportes del Banco Mundial, nota que a medida que las empresas multinacionales ganan familiaridad con el país huésped y con el nivel de ingreso de los consumidores, se reduce la necesidad de tener un socio.

Basándose en un estudio de cincuenta joint ventures en países subdesarrollados, William Dymsza (1986) diferencia a las joint ventures exitosas de las que no lo son, notando que las primeras son aquellas que sobreviven un período de más o menos ocho años y en las que tres entidades- el socio multinacional, el nacional y el gobierno del país huésped perciben que los beneficios son suficientes en relación con los costos. Al gobierno le interesa en primer lugar que gran parte de las ganancias no salgan al extranjero y en segundo lugar, que la firma contribuya al desarrollo del país, garantizando empleo $y$ entrenamiento.

Quisimos saber si la inestabilidad de las joint ventures- tiene algún impacto sobre las personas que trabajan en la firma.

Se analizaron varios tipos de documentos para completar el marco de referencia: catálogos, manuales, organigramas y boletines que publica la empresa, además de artículos de prensa en donde se menciona al joint venture, textos que tratan sobre la cultura corporativa de los sectores que coinciden en la joint venture así como artículos y libros que tratan sobre la situación actual de Venezuela y de la actividad petrolera.

Se utilizaron principalmente entrevistas, pero también observaciones en el sitio. Se realizaron conversaciones extensas con la alta gerencia para conocer la cultura oficial de la empresa, entrevistas semi-estructuradas para conocer las diferencias culturales entre los gerentes ${ }^{3}$ y una pregunta no estructurada para conocer las razones de los cambios observados entre las la empresas de donde provienen e Intesa.

Una vez determinadas las variables a estudiar, se procedió a seleccionar la muestra aleatoria de individuos a los cuales se les aplicó la encuesta ${ }^{4}$. La encuesta está diseñada para detectar la apreciación de los individuos antes y

3 Por razones de tiempo y espacio nos limitamos a entrevistar una muestra seleccionada del total de personas que supervisan o son gerentes en la empresa. Dejamos para una próxima investigación estudiar el punto de vista de los supervisados.

4 Se determinó el tamaño de la muestra utilizando la fórmula propuesta por Scheaffer (1986:58). 
después de la formación de una joint venture.

La directora del Departamento de Cambio Organizacional escribió una carta describiendo los propósitos del estudio y su importancia para la organización. Esta comunicación fue enviada por correo electrónico a cada una de las personas a entrevistar, posteriormente se les llamó para pedirles una cita la cual fue cumplida por casi todos los gerentes sin necesidad de postergarlas. Especialmente los gerentes del interior del país (Amuay, Cardón, Maracaibo, Maturín, Anaco, San Tomé, Puerto La Cruz, Moron y El Palito) cumplieron puntualmente según se había acordado.

Ese mismo departamento proporcionó los teléfonos y direcciones de los departamentos de Recursos Humanos en el interior y en Caracas de manera de localizar a las personas que se debía entrevistar. Se les prometió a los informantes la estricta confidencialidad de la información que ellos iban a brindar.

\section{La joint venture y su cultura corporativa}

En 1997 PDVSA decidió contratar los servicios de informática que antes se hacían en la misma empresa como un esfuerzo por reducir costos y hacer más flexible y de mayor calidad los recursos de informática. Por otra parte PDVSA se dio cuenta que el mercado de servicios tecnológicos había madurado, ofreciendo múltiples opciones en servicios de tecnología de punta.

La empresa conjunta o joint venture (Intesa) se formó en enero de 1997 con aproximadamente 1500 empleados entre
PDVSA y la compañía multinacional SAIC (Science Applications Information Center) con base norteamericana, para suplir los servicios de informática que requiere la industria petrolera.

La alta gerencia de PDVSA contrató a SAIC considerando, además de su experiencia en informática, su cultura corporativa más apropiada para una empresa de informática procurando con ello modificar ciertos aspectos de la cultura de PDVSA, esperando que esto represente una mejoría como empresa comercial.

La mayoría del personal de Intesa, el $90 \%$, proviene de PDVSA. Están, por otra parte, los gerentes que fueron enviados de SAIC para organizar la nueva compañía dentro de los lineamientos de la empresa norteamericana y un tercer grupo proviene del sector privado: empresas de telecomunicaciones, de radio y televisión, financieras, alimentos, quienes trabajan en un edificio aparte donde reside la sede central de Intesa. Estas son personas que fueron contratadas para realizar funciones corporativas como la administración de Recursos Humanos, tesorería, finanzas, etc. Estas funciones, en PDVSA, las realizaba un especialista surgido de la propia compañía.

Intesa se ocupa de dar soporte al usuario. Tiene dos centros de computación para procesar datos y desarrollar todo tipo de software.

Rasgos de la cultura que promueve Intesa: La cultura de Intesa consiste en cinco valores que auspicia la alta gerencia en los programas que ofrece a sus empleados, en los talleres que organiza, en las comunicaciones a través de boletines y en la publicidad que hacen de la compañía. Estos son: la atención al clien- 
te, el ser una organización en continuo aprendizaje (a learning organization), la participación, la ética y el individualismo, valor que promueve a través de los programas de empleado-propietario (employee-ownership) y de los anuncios de vacantes que circulan exclusivamente dentro de la empresa (job-posting).

La atención al cliente: Tanto Intesa como SAIC tienen un compromiso de "permanecer junto a sus clientes" a medida que progresan y se desarrollan. Este trabajo implica cambios de comportamientos porque la mayoría de las personas provienen de PDVSA y tienen poca o ninguna experiencia mercantil. Además, cabe incluir la perniciosa tendencia cultural de poca atención al cliente.

Esta es una debilidad importante de la cual se lamentan de manera consistente las empresas de servicio más variadas. Tal como lo ha observado Granell (1998) las empresas de servicio tienen muchas dificultades para lograr que sus empleados traten con amabilidad y cortesía a los clientes.

Una organización aprendizaje: Intesa enfatiza en sus talleres el hecho de ser una organización aprendizaje (a learning organization), una organización en la cual los errores constituyen oportunidades para aprender, en la que las discusiones de grupo son útiles. De esta manera propicia el manejo abierto de los conflictos.

Una organización que promueve la participación: Esto es algo que se subraya en Intesa y que al mismo tiempo está conectado con el hecho de que Intesa es una organización aprendizaje; se promueve la participación de todos los gerentes y empleados invitándolos a com- partir ideas y no temer a los errores sino aprender de ellos.

El énfasis en la ética: SAIC y ahora Intesa han instituido comités de ética que sirven para vigilar que las transacciones diarias de recursos o información se efectúen sin la intervención de intermediarios. Intesa (y SAIC) cuentan con una hot line (línea telefónica con acceso abierto las 24 horas) en la que cualquier persona de la organización que escucha una conversación acerca de, por ejemplo, un gerente que recibe un favor de un cliente o un proveedor, a cambio de una compra especial, o si un gerente escribe una factura para comprar un computador $y$, en efecto, no lo compra, llama para reportarlo. Todos estos son ejemplos de oportunidades en las que se actúa para mantener la buena reputación.

Programa de empleado-propietario (employee-ownership): Algo que es interesante de mencionar de Intesa es que además de los niveles gerenciales, a todos los empleados se les ofrece la posibilidad de ser propietarios. La filosofía que reside bajo la práctica de empleado-propietario sugiere que mientras más acciones tengan los empleados mayor es su compromiso con la compañía, más satisfechos están con su trabajo, es menos probable que busquen otros empleos y más probable que deseen tener mayor participación accionaria (Rosen et al, 1986).

Los empleados de Intesa se han convertido en accionistas de la empresa en condiciones sumamente ventajosas adquiriendo acciones a crédito, y sin pago de intereses.

El job-posting: Es un instrumento mediante el cual se anuncia a través del correo interno la apertura de una vacante. 
La persona que se siente capaz de ocupar dicha posición y desea cambiar la suya se ofrece para el cargo.

El hecho de que exista el job-posting ha servido para que los gerentes sientan que en Intesa se deben hacer cargo de su futuro profesional y se separen un poco de la tendencia a esperar que la industria petrolera se ocupe de la carrera y del futuro del empleado.

La cultura corporativa y el manejo de los conflictos: La joint venture utiliza la cultura corporativa para atenuar los conflictos que pueden surgir en la organización. La adopción a la cultura corporativa atenúa las posibilidades de que surjan conflictos porque la cultura consiste en un sistema de valores compartidos y algunos están directamente relacionados con el manejo del conflicto, como el énfasis que la empresa hace en la organización de aprendizaje la cual subraya que los conflictos no deben evitarse. Claro está, la organización no logra el manejo perfecto de los conflictos a través de su cultura corporativa. En Intesa, como en otra organización cualquiera, el modo de resolver los conflictos depende de las personalidades que entran en conflicto.

Los conflictos más graves son aquellos que surgen por rechazos a la cultura corporativa y se manifiestan principalmente como intentos por parte de las personas que la rechazan y tratan de disminuir el compromiso organizacional de los demás. Durante el período en que se realizó el estudio se dio, por ejemplo, el caso de un gerente que enviaba correo electrónico a todos los empleados de una manera anónima, explicando que la contribución que hace SAIC a Intesa es míni- ma o nula. Intesa subraya en sus folletos y otras publicaciones la alta estima que siente tanto por su proveedor (SAIC) como por su principal cliente (PDVSA) por lo que la alta gerencia de Intesa mostraba su preocupación por estos correos electrónicos que tanto perjudicaban al clima organizacional de la empresa.

\section{Resultados del estudio cultural en Intesa}

Manejo del conflicto: Para un $51,47 \%$ de las personas que vienen de PDVSA el conflicto se evita tanto en Intesa como en PDVSA y para un $38,24 \%$ se evita más que en PDVSA (Pregunta: si el conflicto se evita o no, ver tabla 1) Entonces, para los de ese sector no hay grandes cambios en ese sentido y si los hay es para un peor manejo de los conflictos.

Se observó que para un $50 \%$ de las personas que vienen de PDVSA no hay cambios en cuanto a la utilidad de las discusiones de grupo para resolver los problemas y para un $39,76 \%$ sirven más las discusiones de grupo para resolver los problemas en Intesa que en PDVSA (Pregunta: si las discusiones de grupo sirven o no para resolver los problemas, ver tabla 1).

Para explicar el que un gran porcentaje de gerentes (que vienen de PDVSA) consideren que los conflictos no se evitan en ninguna de las dos empresas, recordamos a un estudioso del conflicto en las organizaciones (Dalton, 1959). Para Dalton los gerentes están tan ocupados escondiendo el conflicto que al mismo tiempo tratan de resolverlo y pretender que no existe. Especialmente por 


\section{Tabla 1 \\ Cultura Corporativa en una Joint Venture Venezolana Comparación de percepción de los Gerentes}

\begin{tabular}{cccccc}
\hline & Sector de & \multicolumn{3}{c}{$\%$} \\
\cline { 3 - 6 } Categoría de Análisis & Procedencia & Sí & No & $\begin{array}{c}\text { Sin } \\
\text { Cambio* }\end{array}$ & $\begin{array}{c}\text { Sin } \\
\text { respuesta }\end{array}$ \\
\hline \multirow{2}{*}{ El conflicto se evita } & PDVSA & 38.24 & 4.41 & 51.47 & 5.88 \\
& Sector Privado & 0 & 42 & 50 & 8 \\
& Multinacional & 50 & 18.75 & 31.25 & \\
Las discusiones clarifican & PDVSA & 39.76 & 8.82 & 50 & 1.47 \\
problemas complicados & Sector Privado & 33.33 & 17.00 & 50 & 0 \\
& Multinacional & 18.75 & 43.75 & 37.50 & 0 \\
Personas competentes & PDVSA & 4.41 & 52.94 & 38.24 & 4.91 \\
necesitan instrucciones & Sector Privado & 25 & 50 & 25 & 0 \\
precisas & Multinacional & 56.25 & 12.50 & 31.25 & 0 \\
Las descripciones de & PDVSA & 11.76 & 39.71 & 47.06 & 1.47 \\
cargos son útiles & Sector Privado & 25 & 42 & 33 & 0 \\
& Multinacional & 25 & 6.25 & 68.75 & 0 \\
\hline
\end{tabular}

Fuente: Datos propios, según encuesta.

* Respecto a la empresa de origen

lo segundo es muy fácil que los gerentes y/o supervisados vean que los conflictos no se evitan en ninguna de las dos empresas.

Un $50 \%$ de las personas que provienen del sector privado perciben que no hay diferencias en cuanto a si se promueven o no las discusiones de grupo para clarificar los problemas entre Intesa y las empresas de este sector y un $33,33 \%$ considera que las discusiones de grupo sirven más para clarificar los problemas en Intesa que en el sector privado (Pregunta: si las discusiones de grupo sirven o no para resolver los problemas, ver tabla 1).

Un $50 \%$ de las personas del sector privado perciben que en Intesa se evita el conflicto tanto como en las organizacio- nes de las cuales provienen (Pregunta: si el conflicto se evita o no, ver tabla 1). Mientras que un $42 \%$ piensa que se evitan menos que en las organizaciones de donde provienen. Predominan, entonces, las personas de este sector que no ve grandes cambios en ese sentido y si los hay es para un mejor manejo del conflicto $\mathrm{y}$ de las discusiones.

Un $31,25 \%$ de las personas que provienen de la multinacional SAIC percibe que no hay diferencias en cuanto a la frecuencia con la cual se evitan los conflictos entre SAIC e Intesa pero la mitad (un $50 \%$ ) de las personas que provienen de SAIC percibe que en Intesa se evitan los conflictos más que en SAIC (Pregunta: si el conflicto se evita o no, ver tabla 1). 
Un $43,75 \%$ de las personas que vienen de la multinacional SAIC piensan que las discusiones de grupo sirven menos para clarificar los problemas en Intesa que en SAIC; un $37,50 \%$ piensa que no hay cambios entre Intesa y SAIC en cuanto a la utilidad de las discusiones de grupo y un $18,75 \%$ piensa que las discusiones de grupo son más útiles para clarificar los problemas en Intesa que en SAIC (Pregunta: si las discusiones de grupo son útiles o no, ver tabla 1).

Predominan, entonces, las personas que vienen de SAIC que consideran que en Intesa hay un peor manejo del conflicto que en SAIC.

Debido a que en SAIC existe un comité de apelaciones a través del cual se resuelven la mayoría de los conflictos, las personas de esta trasnacional tienen la facilidad de expresar sus quejas o sus molestias a terceros, lo que explica el elevado porcentaje de personas de este grupo que perciben que en Intesa no hay sitio para la resolución de conflictos.

Manejo de la incertidumbre: Más de la mitad de las personas que provienen de PDVSA (52,94\%) sienten que las normas e instrucciones precisas son menos necesarias en Intesa que en PDVSA. Un $38,24 \%$ de las personas de ese PDVSA de ambas organizaciones (Pregunta: si las descripciones de cargos son necesarias o no, ver tabla 1).

A pesar de que un gran porcentaje de personas $(39,71 \%)$ del sector PDVSA nota que se necesitan menos instrucciones precisas en Intesa que en PDVSA la mitad (un $47,06 \%$ ) nota que no hay cambios entre la necesidad de instrucciones precisas que existe en Intesa y en
PDVSA (Pregunta: si las instrucciones precisas son necesarias o no.

Esto se entiende porque los gerentes venezolanos, en general, según Granell (1998) lo ha observado, son personas que están acostumbradas a tener reglas, las necesitan pero también son flexibles y dispuestos a violar las normas y a adaptarse a situaciones nuevas ven grandes cambios en ese sentido.

Un $41,67 \%$ de las personas que provienen del sector privado percibe que las descripciones de cargo son menos necesarias en Intesa que en el sector privado, pero un $33,33 \%$ piensa que las descripciones de cargo son tan necesarias en Intesa como en el sector privado (Pregunta: si las descripciones de cargos son útiles o no, p.32, tabla de resultados).

Un $50 \%$ de las personas que vienen del sector privado consideran que en Intesa es menos necesario dar instrucciones precisas para que una persona competente realice un trabajo apropiado;, un $25 \%$ no ve cambios en este sentido y un $25 \%$ considera menos necesario dar instrucciones precisas en Intesa que en el sector privado (Pregunta: si las instrucciones precisas son necesarias o no, p.32, tabla de resultados).

Un $56,25 \%$ de las personas de la empresa multinacional SAIC perciben que en Intesa es más necesario tener instrucciones detalladas para realizar un trabajo de manera eficiente. Un $31,25 \%$ de las personas de SAIC perciben que en Intesa es menos necesario proveer a un empleado competente de instrucciones precisas para que realice un trabajo de manera eficiente (Pregunta: Las normas 
o instrucciones precisas son necesarias o no, p.32, tabla de resultados).

Por otro lado, un $68,75 \%$ de las personas que proviene de SAIC no ven cambios en cuanto a lo que se refiere a la utilidad de las descripciones de cargos- para ellos estas son igualmente útiles en SAIC como en Intesa. Un $25 \%$ de las personas que provienen de esta firma piensan que las descripciones de cargos son más necesarias en Intesa que en SAIC y un $6,25 \%$ piensa que son menos necesarias en Intesa que en SAIC. (Pregunta: Las descripciones de cargos son necesarias o no., p.32, tabla de resultados).

Aunque con pocas diferencias predominan los porcentajes de gerentes que vienen de SAIC que prefieren dar instrucciones detalladas y descripciones de cargos para realizar un buen trabajo (Pregunta: las descripciones de cargos son necesarias o no).

Ruido en la organización: Los resultados apoyan la idea de que se evita más el conflicto en Intesa que en PDVSA y que en SAIC, mientras que para aquellos que vienen del sector privado se evita menos en Intesa que en el sector privado. También hay ruido porque los tres sectores captan diferentes mensajes acerca del manejo del conflicto en la organización.

El que un porcentaje importante de los que vienen de la multinacional SAIC no ve grandes cambios entre Intesa y SAIC en cuanto al manejo del conflicto se entiende porque para ellos, en ambas organizaciones, los conflictos se resuelven a través de una solicitud de cambio de supervisor a la Junta Directiva de SAIC. También como las personas de que provienen de la firma multinacional tienen mayores posibilidades de aprovechar el instrumento del job-posting (instrumento mediante el cual se anuncia a través del correo interno la apertura de una vacante), sus alternativas de trabajo (en SAIC dentro y fuera de Estados Unidos) son mayores que las de los gerentes venezolanos y pueden atenuar las consecuencias negativas de los conflictos que surgen dentro de la organización.

Para todos los gerentes una relación conflictiva entre supervisor y supervisado se cambia por una relación sana con relativa facilidad si un gerente logra cambiarse de una posición a otra por medio del job-posting, o bien si considera que después de evaluar los beneficios que le produce el ser miembro accionista de la organización, los efectos negativos de la relación conflictiva le resultan ser menores. Pero esto lo logran los que vienen de SAIC con mayor facilidad que los gerentes locales porque tienen mayores alternativas de trabajo.

A pesar del hecho frecuentemente descrito en la literatura (Hofstede, 1980; Granell, 1998; Baird et al, 1990) que se refiere a que los Estados Unidos están entre los países que menos les preocupa la incertidumbre y Venezuela está entre los países que más evitan la incertidumbre, en el presente estudio se observó que en Intesa son los gerentes que provienen de la multinacional los que necesitan mayor número de instrucciones y descripciones detalladas de cargos por lo que son ellos y no los gerentes venezolanos los que peor toleran la incertidumbre. Se ve que hay diferencias en cuanto a la preocupación por la incertidumbre entre las distintas nacionalidades que coinciden en Intesa. Los norteamericanos necesitan reglas porque son enviados de la 
casa matriz y se les exige reportar la realización de su trabajo con detalle mientras que los venezolanos, según lo describe la literatura (Granell, 1998), necesitan reglas pero saben que pueden violarlas si es necesario.

Cuando hay diferencias en la manera como se ve a la organización, se produce un "ruido" que siguiendo a Eileen McEntee (1998) se refiere a cualquier interferencia en el proceso de comunicación entre la fuente y el receptor, que se percibe en el medio ambiente como un gesto que distrae en el receptor, como puede ser la producción de un recuerdo o de un sentimiento de rechazo. También se llama ruido a la diferencia de significado que puede tener un mensaje para las personas, debido a sus diferencias socioculturales o valores (McEntee, 1998). La producción de ruido se traduce en una dificultad en la adopción a lo esencial de la cultura de la empresa.

El ruido determina diferencias entre la cultura corporativa "oficial" y la cultura corporativa "no oficial" que vendrían dadas por dos discursos distintos: el que auspicia la alta gerencia y el que resulta por la interpretación que le da a éste la mayoría. Por un lado la firma promueve un buen manejo del conflicto y una buena tolerancia a la incertidumbre necesarios en una empresa en formación y muy especialmente si se trata de una firma de informática que suelen tener un alto riesgo. Sin embargo, de acuerdo a una suma importante de los tres tipos de gerentes, en Intesa se evaden los conflictos tanto como en la mayoría de las empresas. También, Intesa ha tenido poco éxito en implantar una cultura que favorezca una gran tolerancia a la incertidumbre. Los gerentes norteamericanos encargados de implantar una cultura de alto riesgo se sienten incómodos frente a la poca descripción de cargos y normas aún presentes en Intesa.

Los gerentes que provienen de la multinacional SAIC tienen menores posibilidades de adaptarse a la cultura "no oficial" de Intesa en la cual coexisten rasgos de la cultura de PDVSA y la cultura local. Ellos llegan a conclusiones negativas acerca de los venezolanos. Comentaron que "Intesa se estaba moviendo hacia PDVSA, no hacia la casa matriz". Un gerente atribuía eso a la falta de seguridad y decisión que se derivaba del hecho de desconocer la cultura del país huésped. También se comentó que "Intesa es muy PDVSA", que "los venezolanos están acostumbrados a mucha protección", que "necesitan discutir cada punto miles de veces con lo que se pierde mucho tiempo". Asimismo los gerentes norteamericanos se sentían inseguros ante la costumbre de los venezolanos de violar las normas.

Los gerentes del sector privado venezolano, por otra parte, se sentían excluidos. Esto se corrobora con comentarios frecuentes entre ellos mismos: "a Intesa le preocupa más su cliente (PDVSA) y su proveedor (SAIC) así como también que "Intesa paga mejores sueldos a los de PDVSA y a los de SAIC que a los del sector privado". Estos comentarios se derivan del hecho de que estos gerentes vinieron a ocupar cargos altos y aspiran ganar sueldos más altos que los gerentes de otras firmas locales, es decir, sueldos compatibles con los del sector multinacio- 
nal y con los de un sector que otorga grandes beneficios a sus gerentes (PDVSA).

Para los que vienen de PDVSA era más fácil aceptar a los que vienen del exterior porque los que vienen de PDVSA son la mayoría, no se sienten amenazados por unos cuantos que deben adaptarse a los rasgos culturales de la mayoría. También es importante notar que ambos sectores tienen vías diferentes de transmitir su cultura: unas más eficientes que otras. Para la gran mayoría, los ascensos, la promoción de personal, los salarios se regían por el contrato colectivo de PDVSA (al menos hasta 1999). Es decir, aspectos cruciales de la vida de los gerentes no se modificaron, mientras que SAIC tiene una forma más itinerante de transmitir su cultura corporativa. Ofrece talleres, cursos, emite correos electrónicos, publica boletines que tratan aspectos que resultan de menor importancia cuando se comparan con las posibles amenazas que sienten las personas (bien sea gerentes o supervisados) cuando la organización utiliza las decisiones sobre los ascensos como formas de recibir un premio o un castigo. Estas amenazas propician una adopción de la cultura de PDVSA.

A pesar de que la cultura de PDVSA predomina, los gerentes manifestaron distintas preocupaciones respecto a su convivencia con el sector que proviene de SAIC. Notaban los gerentes que provienen de PDVSA "el trato diferencial que Intesa le da a sus gerentes extranjeros". Como la industria se ha preocupado por ajustarles sus salarios y sus beneficios de acuerdo a la inflación, algunas personas que provienen de PDVSA encuentran que hay diferencia en las condiciones económicas que se le dan a los gerentes extranjeros y venezolanos. Con lo cual se puede concluir que se sienten presionados para competir con el personal gerencial de SAIC. Las expectativas de igualdad en cuanto a salarios contribuyen a desarrollar una mentalidad separatista que refuerza la identificación nacional, dificultando la integración con el sector multinacional.

En la misma línea se puede interpretar un despido importante de 300 personas acaecido en Intesa en 1999 entre los cuales se encontraba parte de la directiva de PDVSA. De acuerdo a lo recogido en las entrevistas éste despido tuvo como efecto un rechazo de la cultura que auspiciaba la directiva de SAIC. A pesar de las promesas que habían sido formuladas a partir de la formación de la joint venture según la cual los que cumplan los requisitos exigidos pueden conseguir empleo en otra sede de Intesa o en otra subsidiaria de SAIC a través del job posting, se observó que los gerentes de PDVSA desarrollaron una desconfianza y suspicacia hacia los de SAIC que se originó al percibir la nueva realidad de los despidos.

El paso del departamento de informática de PDVSA hacia una empresa de negocios y, en particular a una joint venture, explica en gran parte las respuestas a la pregunta abierta ¿ a qué se deben los cambios que se observan entre la organización de la cual provienen e Intesa?, los gerentes respondieron a esa pregunta expresando su preocupación por la inestabilidad laboral.

Entre los gerentes de los tres sectores PDVSA, privado nacional y privado multinacional, se evidenció una inseguri- 
dad con respecto al tiempo que permanecerían en la joint venture. Así por ejemplo, los que provienen de PDVSA comentaban que "hay mucho ruido en el cliente (PDVSA) acerca de que el servicio que Intesa presta es malo". Ruido significa también "las quejas de parte del cliente", en otras palabras cierto rechazo de parte de PDVSA hacia Intesa.

Las quejas de parte de PDVSA como cliente sobre las supuestas deficiencias de Intesa se originaron debido a que usualmente en una joint venture existe el temor de que el proveedor no cumpla con suministrar toda la información necesaria al cliente.

Para los del sector privado los gerentes que provienen de PDVSA no están contentos con el outsourcing decidido por PDVSA. Con ello expresan su sensibilidad hacia el hecho de que sus colegas de PDVSA perdieron estabilidad laboral y también la satisfacción de saber que pertenecían a una de las empresas más importantes del mundo (PDVSA).

Las personas del sector privado notaban que "hay en Intesa una exagerada preocupación por consideraciones presupuestarias", cosa que no veían en las empresas de donde provienen y además, ellos-y no los de PDVSA comentaron acerca de que "habría muchos despidos porque a PDVSA le sale muy cara "Intesa".

La explicación de esta sensibilidad hacia la inestabilidad laboral de los gerentes del sector privado la encontramos en el ambiente en que han crecido las empresas venezolanas. Este fue un ambiente protegido por largas décadas hasta la mitad de los ochenta cuando el cambio hacia un ambiente donde el Estado les quitó la protección exageradas y por supuesto hubo consecuencias, como es el cierre de muchas compañías. Los gerentes que vienen de la empresa privada han observado que los despidos son una de las consecuencias de las crisis.

Los gerentes que provienen de SAIC, por otra parte, sí expresaron su preocupación por lo inestable de sus posiciones en la joint venture ya que la trasnacional no les garantiza un puesto a su regreso a Estados Unidos, pero sus preocupaciones en ese respecto, son mucho menores que las de las personas que tienen como alternativas buscar en el mercado de trabajo nacional.

Para los gerentes que vienen de afuera las noticias sobre recortes presupuestarios son circunstanciales y de una importancia secundaria, mientras que para los del sector privado y los de PDVSA éstos pueden resultar en despidos y en el fracaso de la joint venture. En la agenda de los gerentes de la multinacional tenía prioridad implantar la cultura de SAIC-proyecto que veían con grandes dificultades- por lo menos hasta el momento en que se realizó la investigación, es decir, después de dos años de formada la empresa, tiende a predominar la cultura de PDVSA debido a las condiciones en que se formó Intesa.

Los comentarios de uno y otro sector revelan una permanente amenaza de inestabilidad, a la que muchos no están acostumbrados. No necesariamente los gerentes de Intesa están conscientes de la inestabilidad que caracteriza a las joint venture pero en la oportunidad que tuvieron de expresarse acerca de las diferencias entre Intesa y las organizaciones de 
las cuales provenían, se observó un cierto temor por la incertidumbre que caracteriza sus vidas en la joint venture.

\section{Conclusiones}

Impacto de las diferencias culturales en Intesa: El estudio que se describe en estas páginas corrobora algunas de las investigaciones previas realizadas en joint venture. Estas notan que las diferencias culturales que existen entre los socios dificultan la implantación de la cultura oficial (aquella que auspicia los directivos de la empresa). Muestra que las diferencias en cultura nacional y/o en cultura corporativa influencian la manera como los integrantes de la organización absorben importantes aspectos de la cultura corporativa de la joint venture.

El presente estudio permite entender cómo el paso de una empresa gubernamental a una empresa de negocios fue además de rápido, inesperado. Esta transición fue difícil porque entre los "viejos" empleados se escuchaban comentarios acerca de la incertidumbre que caracterizaba sus vidas en la nueva empresa, ya que sabían que muchos ya no eran necesarios en la nueva empresa que contaba con procesos tecnológicos más sofisticados. Esto hizo que se apegaran a su viejas normas y desarrollaran fuertes resistencias a la nueva cultura. En Intesa sucedió algo similar con un gran número de empleados que provenían de PDVSA por lo que la alta directiva decidió despedir a un número importante de ejecutivos que representaban a la vieja cultura.

La cultura corporativa de Intesa: Los resultados de esta investigación constatan la importancia de las condicio- nes iniciales en las que se forma una joint venture al determinar que una cultura predomine sobre otra. Estas condiciones son: la ubicación, las instalaciones, el lenguaje utilizado en la joint venture, los horizontes de carrera, el que la joint venture dependa mucho de sus socios. Las condiciones iniciales, es decir, el personal que predomina en Intesa proviene de PDVSA, las instalaciones son mayormente las de PDVSA, el lenguaje (español) que hace que los que provienen de PDVSA sientan pocos cambios en la comunicación en la organización; y las condiciones contractuales (los pagos y otras condiciones de trabajo se regían mayormente por el contrato laboral de PDVSA hasta inicios del 1999), determinan que las reglas de conducta que prevalecen en la firma sean más familiares a PDVSA que a SAIC o al sector privado.

Este estudio muestra un elemento más que contribuye al predominio de una cultura sobre otra: las vías que posee cada sector de transmitir su cultura corporativa. Estas vías no son igualmente efectivas. PDVSA dicta las sanciones y castigos porque la mayor parte del personal de Intesa se regía por el contrato laboral de PDVSA. La aceptación de la cultura corporativa depende de las oportunidades de ascenso que ofrece la firma y los gerentes aprenden esas oportunidades observando las carreras de los otros así como lo que se premia y se sanciona en la firma. Por esta razón el contrato laboral y las vías de ascenso constituyen una vía muy poderosa de transmitir las pautas y normas que se deben seguir en la firma y de lograr que se cumplan.

En contraste, la alta gerencia encargada de transmitir la cultura de la mul- 
tinacional SAIC, transmite su cultura corporativa a través de talleres y publicaciones. Estas son vías itinerantes y tratan de aspectos más superficiales cuando se les compara con factores que afectan los ascensos dentro de la organización.

La gran mayoría de personas que provienen de PDVSA sienten que hay pocas diferencias entre Intesa y PDVSA. En las respuestas a la pregunta abierta " $¿ A$ qué se deben los cambios entre Intesa y PDVSA?" explicaron que observan pocos cambios entre ambas firmas. Notan, por ejemplo, que para contactar con los niveles de jerarquía pueden tener igual facilidad de acceso en Intesa como en PDVSA. En la nueva organización trabajan sobre todo personas que vienen de la industria petrolera con la que se relacionan de manera muy similar a la que venían haciéndolo anteriormente. Y los que provienen del sector privado y el multinacional ven que Intesa se caracteriza por un alto grado de paternalismo. Para ellos este rasgo permanece debido a la herencia de una cultura jerárquica y paternalista como la de PDVSA.

La multinacional SAIC era vista como una empresa de gran prestigio, pero esto no servía para contrarrestar las condiciones iniciales en las que se formó Intesa. Aunque éste no fuera el propósito de los gerentes extranjeros quienes venían para implantar la cultura de SAIC. En principio su cultura se adaptaba a las necesidades de la firma en momentos en los que necesitaba pasar de una empresa estatal a una empresa de negocios y específicamente de informática. Ciertos rasgos de la cultura de PDVSA están muy marcados en la cultura de Intesa. Comentaban los gerentes que venían de
PDVSA, por ejemplo, que "Intesa es un departamento más de PDVSA" y los de SAIC que "Intesa es muy PDVSA".

A pesar de que la alta gerencia creía que su estructura y su sistema de incentivos ayudaría a promover una identificación con la joint venture, surgió una diferenciación entre tres grupos sociales y los miembros de un grupo terminaron excluyendo a los miembros del otro. Aún con los intentos de la alta gerencia en implantar una cultura aceptada por todos los miembros, estos se agruparon identificándose con sus identidades nacionales o corporativas.

Se observó, entonces, que hay integración cultural en lo formal pero en la práctica hay un costo que implica un sacrificio por parte de los tres sectores que coinciden en Intesa; a los cuales se le dificulta la adaptación a la cultura "oficial" (la que trata de implantar la alta gerencia) de Intesa.

Inestabilidad de las joint venture e inestabilidad laboral: Se encontró una correlación entre la inestabilidad que caracteriza a las joint venture y una preocupación por la inestabilidad laboral que recurrió como tema en casi todas las entrevistas. Los temores surgen porque existe la posibilidad de que la joint venture no sea una alternativa favorable o viable para los socios.

Los gerentes que vienen de PDVSA (la mayoría en Intesa) expresaron su satisfacción con el hecho de que ahora son una empresa de negocios y no una empresa estatal: de mayor apertura en las discusiones, horizontes de carrera que estimulan la iniciativa individual, la cual en principio ofrecen mejores oportunidades de avanzar pero se quejaron de la inestabilidad laboral que sienten en la joint venture.

La inestabilidad laboral que amenaza a los gerentes está asociada con 
dos hechos: primero, a la inestabilidad que caracteriza a este tipo de empresa y segundo, el paso de una empresa estatal a una empresa privada. De los dos factores asociados con la inestabilidad laboral, el primero es el más importante, pues los tres grupos de gerentes sienten inestabilidad, es decir, también los gerentes que provienen de empresas de negocios (sector privado y SAIC) sienten más inseguridad que en los trabajos de donde provienen.

Pero esto no quiere decir que Intesa se va a deshacer, hoy por hoy los hechos muestran que esto no será así. Las tendencias en el 2001 indican que Intesa está creciendo con PDVSA y SAIC como socios buscando clientes en otros países en Latinoamérica. Aunque entre los objetivos de nuestra investigación no estaba el encontrar una correlación entre las diferencias culturales y la duración de la joint venture, no se encontraron en las entrevistas indicios de que las diferencias culturales tengan incidencia en la duración de la joint venture. No se comprobó que un problema antropológico tiene necesariamente que resultar en el rompimiento de una sociedad. Una decisión de tal magnitud obedece eminentemente a razones financieras.

\section{Bibliografía Citada}

Baird, Inga; Lyles, Marjorie y Wharton, Robert (1990). Attitudinal differences between American and Chinese manageers regarding joint venture management. Management International Review Special Issue, 53-68.

Barkema, Harry y Vermeulen, Freek (1987). What differences in the cultural background of partners are detrimental for international joint ventures? Journal of International Business Studies Fourth Quarter, 845-864.

Beamish, Paul (1988). Multinational joint ventures in developing countries London: Routledge Books.

Berlin, Margalit (1986). Sewing compliance: State policies, work and housing for the female immigrant in Venezuela Ph.d. dissertation for Columbia University Anthropology Department.

Berlin, Margalit (1994). Cultura corporativa y entorno de negocios. Trabajo de ascenso para la categoría de Profesor Asociado Universidad Simón Bolívar.

Berlin, Margalit (1995). "Accesing organizations: Investigating attitudes towards technology". Technology Studies 2: 380-396.

Berlin, Margalit (1996). "Business environment and corporate culture in Venezuela". Organization Studies 17, 5, 843-855.

Berlin, Margalit (1999). El rol de la cultura corporativa en una empresa multinacional. Revista Venezolana de Gerencia 4 , 8 Agosto, 67-82.

Berlin, Margalit (2000). Cultura corporativa en una joint venture: El caso de Intesa. Trabajo presentado para ascender a la categoría de Profesor Titular Universidad Simón Bolívar, Caracas.

Cartwright, Susan y Cooper, Gary (1989). Predicting success in joint venture organisations in information technology. Journal of General Management 15, 1 Autumn, 39-47.

Contractor, Farok and Lorange Peter (1986). Why should firms cooperate? The strategy and economics basis for cooperative ventures. In Contractor, Farok and Lorange, Peter (ed.) Cooperative strategies in international business: Joint ventures and technology 
partnerships between firms (pp.4-28) Mass, Lexington: Lexington Books.

Dalton, Melville (1959). Men who manage: Fusions of feeling and theory in administration. New York: Wiley.

Dymsza, William (1986). "Successes and failures of joint ventures in developing countries: Lessons from experience". En: Contractor, Farok y Lorange, Peter op. cit. 403-424.

Giddens, Anthony (1999). Un mundo desbocado: Los efectos de la globalización en nuestras vidas. Editorial Taurus. Madrid.

Gómes-Cáceres, Benjamin (1988). "Joint venture cycles: The evolution of ownership strategies of U.S.MNs., 1945-75". En: Contractor, Farok y Lorange, P. op. cit. (pp.111-128).

Granell, Elena (1998). Exito gerencial y cultura. Ediciones IESA. Caracas.

Harrigan, Kathryn (1986). "Strategic alliances and partner asymmetries". En Contractor and Lorange, op. cit. pp. 205-226.

Hendry, John (1999). "Cultural theory and contemporary management organization". In Human Relations 52, 5, 557-577.

Hofstede, Geert (1980). Culture consequences: International differences in work related values Beverly Hills CA Sage Publications.

Inkpen, Andrew y Beamish, Paul (1997). Knowledge, bargaining power and the instability of joint ventures. Academy of Management Review 22,1, 177-202.

Kanter, Rosabeth Moss (1989). Becoming palls: Poling, Allying and liking accross companies Academy of $\mathrm{Ma}$ nagement Executive August, 183-189.

Kanter, Rosabeth Moss (1994). Do cultural differences make a business difference? Contextual factors affecting cross-cultural relationship success Journal of Management Development 13, 2, 5-23.

Killing, Peter (1983). Strategies for joint venture success. Crown Hellin Publishers. London.

Kogut, Bruce (1988). The effect of national culture on the choice of entry mode Journal of International Business Studies 19, 3, 41-32.

Kunda, Guideon (1992). Engineering culture: Control and commitment in a hightech corporation Philadelphia: Temple University Press.

Lam, Alice (1997). Embedded firms, embedded knowledge: Problems of collaboration and knowledge transfer in global cooperative ventures Organization Studies 18/6, 973-996.

Lei, David y Slocum, John (1991). The cultural diversity of western conceptions of management International Studies of Management and Organization XIII, 1-2- Spring- Summer, 75-96.

Leung, Kwok; Smith, Peter y Zhongming, Wang (1996). Job satisfaction in joint venture hotels in China: An organizational justice analysis Journal of International Business Studies Special Issue.

McEntee, Hielen (1998). Comunicación intercultural: Bases para la comunicación efectiva en el mundo actual México: McGraw Hill.

Morris, Michael; Williams, M.; Leung, K.; Larrich, R. Mendoza, T.; Bathnagar, D.; Li Kondo, M. y Hu, J. (1998). Conflict management style: Accounting for cross national differences Journal of International Business Studies 29, 4, Fourth Quarter, 739-748.

Newman, Oscar (1992). Birth of a succesful joint venture Boston: University Press of America. 
Ogliastri, Enrique; et al (1999). Cultura y liderago organizacional en 10 países de América Latina. Academia Revista Latinoamericana de Administración \#22, Primer semestre de 1999.

Park, Seung y Russo, Michael (1996). When competition eclipses cooperation: An event history analysis of joint venture failure Management Science 42, 6, June, 875-890.

Park, Seung y Ungson, Gerardo (1997). The effect of national culture, organizational complementarity and economic motivation of joint venture dissolution. Academy of Management Journal 40,2,279-307.

Rodner, James-Otis (1992). "Los convenios de empresa conjunta ("joint venture") y la legislación venezolana". Revista del Colegio de Abogados del Distrito Federal 151 Julio-Diciembre, 7-81.

Rodner, James-Otis (1993). La inversión internacional en países en desarroIlo. Editorial Arte. Caracas.

Rodríguez, Carlos (1996). The richness of being different....The challenge of working together: Bonding and commitment in strategic alliances: A latent variant structural modeling approach En: Leal, Ricardo and Husted, Bryan (eds.) Proceedings of the Business Association for Latin American Studies Monterrey, México.

Rodríguez, Carlos (1997). Convergent management styles and intercultural fit: The role of culture in international strategic alliances USA_Mexico En: Gertner, G., Bocater, P.F. and Leal, Ricardo (1997) Proceedings of the Business Association for Latin American Studies, Río de Janeiro.

Rosen, Corey y Young, Karen (1986). Employee ownership in America: The equity solution Lexington, Mass.: Lexington Books.
Salk, Jane (1993). Behind the state of the union: The impact of design and social process on relationships in shared management joint venture teams. Report summary North Carolina: Duke University.

Sathe, Vijay (1985). Culture and related corporate realities: Text, cases and readings on organizational entry, establishment and change Illinois: Richard Irwin, Inc.

Schein, Edgar (1985). Organizational culture and leadership San Francisco: Jossey Bass Editors.

Swan, Peter y Etlie John (1997). U.S. Japanese manufacturing equity relationships. Academy of Management Journal 40,2, 462-479.

Valdés Llaneza, Ana y García, Canal (1998). "Distinctive features of domestic and international joint ventures". Management International Review 38, 49-66.

Vernon, Raymond y Wells, Louis (1991). The manager in the international economy. Englewood Cliffs, New Jersey: Prentice Hall.

Wong, Yim Yu; Maher, Thomas; Jenner, Richard; Apple, Allen y Hebert, Len (1999). Are joint ventures loosing their appeal in China? SAM Advanced Management Journal Spring, 4-20.

Wright, Mike; Hoskisson, Robert; Filatotchev, Igor y Buck, Trevor (1998). Revitalizing privatized Russian enterprises Academy Of Manament Executive $12,2,74-85$.

Zeira, Yoram; Newbury, William y Yeheskel, Orly (1997). Factors affecting the effectiveness of equity international joint venture Management International Review 37, 3, 259-279.

Zhuang, Louis; Ritchie, R. y Zhang, Q. (1998). Managing risks in China Long Range Planning 31, 4, August, 606-614. 